\title{
Stochastic Adsorption of Diluted Solute Molecules at Interfaces
}

\author{
Jixin Chen* \\ Department of Chemistry and Biochemistry, Nanoscale and Quantum Phenomena Institute, Ohio University, Ath- \\ ens, $\mathrm{OH} 45701$.
}

KEYWORDS: Single-Molecule Diffusion, Theory of Single-Molecule Adsorption, Langmuir Adsorption,

Ward-Tordai Equation

\begin{abstract}
Here the diffusion and the stochastic adsorption of single diluted solute molecules on flat and patterned surfaces are analytically solved by integrating the single-molecule probability density function (PDF) of Fick's $2^{\text {nd }}$ law of diffusion. The solution is then compared to ensemble solutions such as the Langmuir-Schaefer equation and the Ward-Tordai equation. The equation is then corrected with several Monte Carlo random-walk simulations of model systems, namely diffusion of bulk solute molecules to a flat bouncing surface, an imaginary flat interface, and a flat adsorptive surface. In the short-time limit with a correction, the method reproduces the same result of the Langmuir-Schaefer equation. In the long-time limit, the back-diffusion term of the Ward-Tordai equation is obtained via simulation and explained. On the bouncing surface, a fixed solute-surface collision rate is observed for a given integration time (the time of each measurement cycle). However, the rate is dependent on the integration time because of the multiple collisions of the same molecules over different time regions as such a characteristic integration time to represent the upper limit of the mass flux is derived. The simulation also clarifies that the factor of two correction of the single-molecule equation is due to the missing of the self-mimetic fractal nature of diffusion in the single-molecule PDF at a given time. Also, due to the mirror effect, the single-molecule equation should be multiplied with a factor of four for an imaginary interface, which offers a way to predict the diffusive collision frequency in bulk solutions. For the adsorptive surface, the power-law decay function over time of adsorption in the Langmuir-Schaefer equation is visualized during the simulations to come from an evolving concentration gradient near the surface along with the depletion of the bulk solute molecules near the surface. Finally, simulations on self-assembled monolayer formation with the Langmuir isotherm model are used to illustrate the limitations of the various methods.
\end{abstract}




\section{INTRODUCTION}

Diffusion, ${ }^{1,2}$ adsorption, and absorption of single molecules inside a confined volume are fundamentally interesting in many fields such as cell biology, ${ }^{3-5}$ biosensing, ${ }^{6,7}$ separation, ${ }^{8}$ fluidic dynamics, ${ }^{9}$ reaction kinetics, ${ }^{10}$ catalysis, ${ }^{11,12}$ and batteries. ${ }^{13}$ For example, stochastic absorption of antibody and antigen is responsible for the immune response of a living cell. Absorption of a single messenger molecules triggers motions of ion channel proteins on a cell membrane which is an important function to exchange materials and information among cells such as muscles and neurons. ${ }^{14}$ Stochastic adsorption of single molecules on surfaces is responsible for many separation techniques such as chromatography, membrane filtration, and heterogeneous catalysis. ${ }^{15-17}$ The stochastic collision of molecules in a diluted solution is the key process for reaction kinetics. Biosensing and lab-on-a-chip synthesis are often realized by molecular diffusion. With the development of single-molecule detection methods, ${ }^{6,7,18-20}$ there is a need to simplify the diffusion equations to estimate the order of magnitude of simple parameters such as collision rates. In this article, I am particularly interested in molecules or colloids diffusing in a solution and adsorbing on a surface.

Diffusion has been empirically summarized by Fick's laws of diffusion. ${ }^{1,2}$ For example, the diffusion of materials from a high-concentration reservoir through an interface into the bulk volume of interest is described by a time- and space-dependent concentration gradient function $C(x, t)$, which is summarized into the 1D diffusion equation that obeys the Fick's second law of diffusion: ${ }^{1,2}$

$$
\frac{\partial C(x, t)}{\partial t}=\frac{\partial}{\partial x}\left(D(x, t) \frac{\partial C(x, t)}{\partial x}\right)
$$

where $t$ is time (unit s), $x$ is the distance away from the interface $(\mathrm{m}), C(x, t)$ is the concentration gradient (molecules $\left.\mathrm{m}^{-3}\right)$, and $D(x, t)$ is the time-and-space-dependent diffusion coefficient $\left(\mathrm{m}^{2} \mathrm{~s}^{-1}\right)$, a constant if the time and space-dependent are negligible under certain conditions.

In a special case for our interested molecules diffusing in a homogeneous bulk solution, when $D(x, t)$ is averaged into a global constant $\langle D\rangle=D$, the equation resembles the heat equation formulated by Joseph Fourier in 1822 to describe heat conduction in materials ${ }^{2}$ which are phonon diffusion:

$$
\frac{\partial f}{\partial t}=\frac{\partial^{2} f}{\partial x^{2}}
$$

where $f$ is the heat distribution along, say, a 1D rod with a hot end and a cold end.

This special case has a formal analytical solution of the concentration over space and time: ${ }^{1}$

$$
C(x, t)=\frac{1}{\sqrt{4 \pi D t}} e^{-\frac{x^{2}}{4 D t}}
$$

where $C(x, t)$ (unit $\left.\mathrm{mol} / \mathrm{m}^{3}\right)$ is the concentration of the molecules at space $x(\mathrm{~m})$ from origin $x=0$ and time $t$ from initial time $t=0$ that all molecules are at the origin. Equation 3 is a 1D Gaussian distribution function with the standard deviation $\sigma=\sqrt{2 D t}$, and is normalized to the unit in all $x$ space, i.e. at any giving snapshot of $t, A \int_{-\infty}^{\infty} C(x, t) d x=1$ (mole) where $A$ is a unit area. The Gaussian distribution is an approximation of the van Hove correlation function for the Brownian diffusion. ${ }^{21,22}$ To make the molecular unit work, the equation assumes an area of $A=1$ unit length ${ }^{2}\left(\mathrm{~m}^{2}\right)$ which is hidden in the perpendicular dimensions, i.e., the infinitesimal volume along the box (or cylinder) will be $A \mathrm{~d} x\left(\mathrm{~m}^{3}\right)$.

The diffusion constant $D$ for a colloid, an organic molecule, or a protein in a liquid can be estimated by the Stokes-Einstein equation: ${ }^{23,24}$

$$
D=\frac{k_{B} T}{6 \pi \eta r}
$$


Where $k_{\mathrm{B}}$ is the Boltzmann constant, $T$ is temperature, $\eta$ is the viscosity of the solution, and $r$ is the radius of the particle. For a molecule approximated to a small ball, $r$ can be estimated from the molecular weight $M w=\frac{4}{3} \pi r^{3} \rho\left(\mathrm{kg} / \mathrm{m}^{3}\right)$, where $\rho$ is the density of the neat molecule in the solid or liquid state. All SI units.

An analytical solution of the adsorption kinetics of diluted diffusive molecules onto a flat surface was firstly reported by Ward and Tordai in a 1946 paper by integrating the diffusion equation: ${ }^{25}$

$$
\left.\Gamma(t)=2 c_{b} \sqrt{\frac{D}{\pi}} \sqrt{t}-\sqrt{\frac{D}{\pi}} \int_{0}^{\sqrt{t}} \frac{c(\tau)}{\sqrt{t-\tau}} d \tau\right)
$$

where $\Gamma$ (unit $\mathrm{mol} \mathrm{m}^{-2}$ ) is the number of molecules adsorbed on a unit area of the surface, $t$ is the time since the formation of the fresh surface (s), $D$ is the diffusion constant $\left(\mathrm{m}^{2} \mathrm{~s}^{-1}\right), c_{\mathrm{b}}$ is the bulk and $c(\tau)$ is the sub-surface molar concentration of the solute in the solution $\left(\mathrm{mol} \mathrm{m}^{-3}\right)$, and $\tau$ is a dummy variable with the unit of time (s). The first part of the equation is the Langmuir-Schaefer Equation reported 1937 suitable for the short-time limit, ${ }^{26}$ and the second half of the equation is so-called the "back-diffusion" of molecules from the surface over a longer time, i.e. a correction to the desorption and self-repulsion of the molecules. We can picture this equation into an adsorption isotherm curve rises fast at the beginning dominated by the first term, slow down, and saturate over time because of the growth of the second term.

This ensemble approach is mathematically complicated involving fraction calculus and Laplace transform of convolution and mechanistically confusing, e.g., the square-root-dependent growth of the first term is unusual in kinetics, the physical meaning of the time-dependent sub-surface concentration is not clear and the whole back-diffusion term is confusing. ${ }^{27,28}$ The sub-surface concentration is often been extracted from the experimental data which makes the equation semi-empirical in many cases. Specifically, the Ward-Tordai equation is often used to measure the effective diffusion constant from the experimental data ${ }^{25,29}$ which beats the purpose of using the Stoke-Einstein equation to predict the adsorption kinetics. Besides, it is difficult to extract the underlying adsorption mechanism from the effective diffusion constant that is often several orders of magnitude different from the value predicted by the StokeEinstein equation. ${ }^{25,29}$ Thus, it has been an ongoing challenge to simplify the calculations and clarify the meanings. ${ }^{27-33}$ These complexities have limited the applications of the Ward-Tordai equation (Equation 5) in many fields such as chemistry, biochemistry, biophysics, biotechnologies, and chemical engineering. ${ }^{27,28}$ In these fields, the diffusive adsorption is a critical process yet a simple-enough-to-understand model to describe the stochastic single-molecule behavior is still lacking, as far as I know from searching the literature and talking to many peers in the biophysics field. In this report, I am targeting the adsorption problem using a single-molecule approach that is intuitive to understand instead of the well-established ensemble approach. As a major motivation, once the single-molecule method is established, equations to calculate single-molecule collision frequencies among each other and to a surface with any shape and curvature in both semi-infinite and confined space can be derived in the future.

\section{RATIONAL}

The 1D diffusion distribution of many molecules, Equation 3, also represents the diffusion probability density function (PDF) of any molecule in any dimension inside the media. ${ }^{1}$ Giving no media flow and symmetric in the media structure, it is natural to assume that the molecule inside the media will diffuse symmetrically over any given direction, i.e., its PDF over time is a diffusion ball and symmetric. In the special case with an infinite area of the interface as we have assumed for Equation 3, the diffusion of the molecules in the lateral direction, the lateral fraction of the PDF, cancel with those of other molecules, i.e., the molecules switch positions and maintain the statistical distances in the media. As such, only the diffusion probability that is perpendicular to the interface survives for the measurement of the 1D gradient. We need two assumptions to extend the ensemble distribution to single-molecule PDF: (1) assuming all 
molecules are equal for a normal diffusion, ${ }^{4}$ i.e., the ergodic hypothesis in thermodynamics, and (2) no inter-solute interaction, or the concentration difference over the space does not change the intermolecular interaction among the solutes, i.e., under the diluted condition.

Thus, for single-molecule and single-particle diffusion, or Brownian motion, assuming all 3D directions are symmetric and inherit the ensemble diffusion constant, the probability density function (PDF) becomes

$$
p(x, y, z, t)=\left(\frac{1}{4 \pi D t}\right)^{3 / 2} e^{-\frac{x^{2}+y^{2}+z^{2}}{4 D t}}=\left(\frac{1}{4 \pi D t}\right)^{3 / 2} e^{-\frac{\vec{R}^{2}}{4 D t}}
$$

where the vector $\vec{R}$ representing the distance and the angles to the origin position of the molecule, at $t=$ $0, x_{0}=y_{0}=z_{0}=0$ for ease of expression. Equation 6 pictures the symmetric diffusing ball with the maximum at the center, Gaussian decaying over all directions, and normalized to one unit if integrated over all volume.

\section{tion 3:}

The 1D PDF of single-molecule away from an origin has the same format as the ensemble Equa-

$$
p(x, t)=\frac{1}{\sqrt{4 \pi D t}} e^{-\frac{x^{2}}{4 D t}}
$$

where the diffusion constant $D$ is the same value from the ensemble measurement, $t$ is time, and $x$ is the distance from the origin interface. Equation 7 works if there is a large-area (semi-infinite) interface perpendicular to the direction of interest, or in a confined rod space where the sidewalls just simply bounce the molecules back. The SI unit of $p$ is $\mathrm{m}^{-1}$, with hidden area $\mathrm{m}^{-2}$ in the perpendicular dimensions, i.e. the probability is normalized to one unit in the cylindrical volume with $1 \mathrm{~m}^{2}$ area and semi-infinite length.

This restriction is satisfied in many interesting questions to calculate the frequency of solute molecules diffusing to a target area on a flat surface, e.g. self-assembled monolayer, and typical biosensing platforms such as probes binding to immobilized protein or DNA on a flat surface. For example, we have measured the absorption rate of dye-DNA staining using single-molecule fluorescence microscopy which triggers the interest for this report. ${ }^{34}$

The accumulated number of molecules collide on the surface can be calculated by assuming that the probability of any solute molecule hitting a nearby surface is its error function in the $z$ dimension (perpendicular to the surface). A scheme is shown in Fig. 1. Integrating all molecules in the solution in the cylindrical volume of interest yields the most probable number of molecules (\#) hitting the surface at a given time $\Delta t:^{34,35}$

$$
\Gamma(\text { in } \Delta t)=\int_{z=0}^{\infty} C A \mathrm{~d} z \frac{\int_{x=z}^{\infty} e^{-\frac{x^{2}}{4 D \Delta t} \mathrm{~d} x}}{\int_{x=-\infty}^{\infty} e^{-\frac{x^{2}}{4 D \Delta t}} \mathrm{~d} x}=\int_{z=0}^{\infty} C A \mathrm{~d} z \int_{x=z}^{\infty} \frac{1}{\sqrt{4 \pi D \Delta t}} e^{-\frac{x^{2}}{4 D \Delta t} \mathrm{~d} x}
$$

where $z$ and $x$ are the lengths of space shown in Fig. 1A (unit $\mathrm{m}$ ), $A$ is the area of interest on the surface (unit $\mathrm{m}^{2}$ ), $A \mathrm{~d} z$ is an infinitesimal volume in the solution of interest, $C$ is the number concentration of the solute molecule in the solution (unit \# $\mathrm{m}^{-3}$ ), thus, $A C \mathrm{~d} z$ is the number of molecules in the volume $A \mathrm{~d} z$ that is $z$ (unit $\mathrm{m}$ ) away from the surface, $D$ is the diffusion constant of the molecule (unit $\mathrm{m}^{2} / \mathrm{s}$ ), and $\Delta t$ is the elapsed time (unit s). 


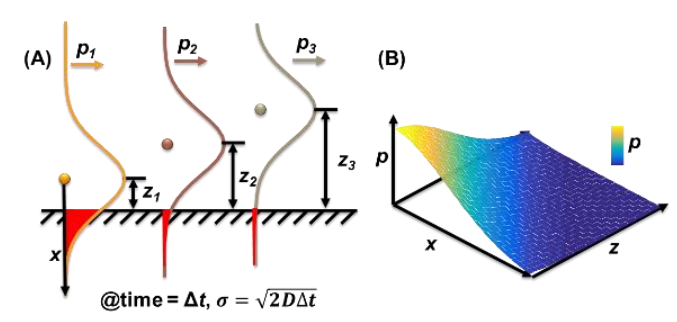

Fig. 1. (A) Snapshot of particles (dots) showing at their origins and scheme of their 1D diffusing PDFs (Gaussian) over space at time $\Delta t$ (curves). The probability of each particle hitting an interface perpendicular to its diffusing direction is shown in the red-colored error functions. (B) Scheme of integrating all the error functions in Equation 8.

Solving Equation 8 (Fig. 1B) yields a solution that the accumulated number of molecules colliding to the surface area of $A$ within time $\Delta t$ is

$$
\Gamma(\text { in } \Delta t)=A C \sqrt{\frac{D \Delta t}{\pi}}
$$

which has been erroneous integrated before ${ }^{34}$ and is corrected here.

Comparing to the Langmuir-Schaefer equation, ${ }^{26}$ major problems of Equation 9 is missing a factor of two for it, and $\Delta t$ instead of the elapsed time $t$. Missing the back-diffusion term of the Ward-Tordai equation is expected since no surface reaction model has been included and we will address it later with a model of a self-assembled monolayer. An additional question arises from Equation 9 is about the average single-molecule hitting rate $\left(\mathrm{s}^{-1}\right)$ counted at $\Delta t$ time resolution (by averaging number of hits observed in each time step):

$$
\langle r\rangle=\frac{\Gamma(\text { in } \Delta t)}{\Delta t}=A C \sqrt{\frac{D}{\pi \Delta t}}
$$

where rate $\langle r\rangle$ is the average number of molecules hitting per second anywhere in the whole area $A$.

Equation 9 has some elements of the Ward-Tordai equation and should be corrected to obtain consistency. Equation 9 suggests that the rate measured is a function of observation time $\Delta t$, i.e., the hitting rate counted in $1 \mathrm{~ms}$ and $1 \mathrm{~s}$ is $\sim 33$ times different, but intuitively it should be the same value that is independent on the counting time interval. Thus, justification and explanation are required, for which, I run a Monte Carlo numerical simulation ${ }^{35}$ using MATLAB with a random walking model ${ }^{24,36}$ to investigate what is happening under some experimental conditions. Two major suspects causing the problem of my approach are the fractal nature of the diffusion, ${ }^{37-39}$ and the complicated PDF at the surface caused by the mirror effect.

\section{METHODS}

The Monte Carlo simulation is carried out on a laptop equipped with an Intel i7 CPU (2.2 GHz) and $16 \mathrm{~Gb}$ of memory. A basic version of MATLAB 2014b with no toolbox is used for all simulations, i.e., single-CPU is used for all simulations. I use a previously coded fitting algorithm jcfit ${ }^{40}$ (Github) to fit the curves. The two major random functions to generate the step motion of each molecule are from 
MATLAB, rand () creating evenly distributed random numbers and randn () generating Gaussian distributed random numbers. Detail parameters and settings for the simulations are listed with the simulation results in the later sections. The single-molecule diffusion of the solute molecules in confined semi-infinite volumes and their collisions to the walls are simulated. Inter-molecular collisions are not simulated. Detail simulation parameters are shown in figure captions and associated text. Example source codes to generate 1D single-molecule displacements are included in the supporting information. Typical computer time used for the simulations is from a few seconds to a few hours.

\section{RESULTS AND DISCUSSION}

\section{Monte Carlo control simulation of $1 D$ diffusion}

I first test the random function $r n d n()$ in MATLAB with a control simulation in generating time-dependent diffusion profiles with a $1 \mathrm{D}$ random walk model. I place 10,000 molecules in the origin of a $1 \mathrm{D}$ space at $t=0$, then pick a time step $\Delta t=1 \mathrm{~ms}$ and let the molecules walk either direction at each time step creating a movie with $1 \mathrm{~ms}$ frame resolution. The walking distance of each step is Gaussian distributed with the standard deviation or diffusion root mean square displacement $\mathrm{RMSD}=\sqrt{2 D \Delta t}$, where $\mathrm{D}$ is calculated with Equation 4 assuming $T=300 \mathrm{~K}, \eta=8.9 \times 10^{-4} \mathrm{~Pa}$ s (media is water), the molecule is assumed a ball with molecular weight $1271 \mathrm{~g} / \mathrm{mol}$ and molecular density $0.8 \mathrm{~g} / \mathrm{mL}$ mimicking an organic dye molecule. Thus, $D=2.88 \times 10^{-10} \mathrm{~m}^{2} \mathrm{~s}^{-1}$, and the RMSD $\sigma=\sqrt{2 D \Delta t}=760 \mathrm{~nm}$. Fig. 2 plots selected histograms of the locations of these molecules spread out over time at $t=n \Delta t$ where $n=1,2,3, \ldots, 10^{5}$, with a total length of simulation $100 \mathrm{~s}$. The histograms are then compared with the Gaussian probability density function (PDF) curves directly calculated from Equation 7. The agreement between the results of the stepwise simulation and the calculated PDF confirms that the random function and the random walk model works correctly to simulate a Brownian diffusion within the time of interest from milliseconds to minutes.

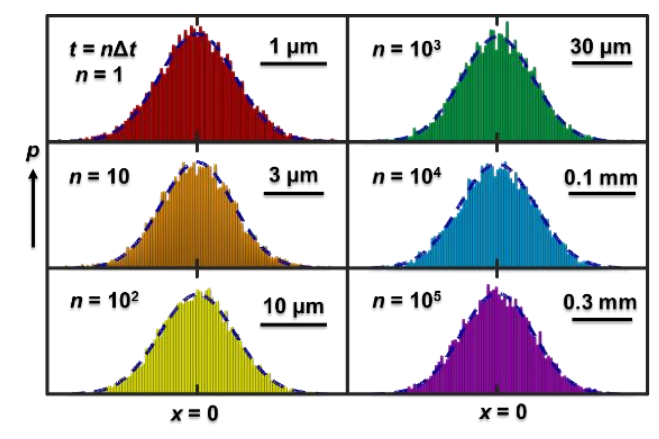

Fig. 2. Histograms of simulated $1 \mathrm{D}$ diffusion of $10^{4}$ molecules that have been placed at the center position $x=0$ at $t=0 \mathrm{~ms}$ then each randomly walks along $x$ creating a concentration gradient at $t=n \Delta t$. The diffusion constant $D=2.88 \times 10^{-10} \mathrm{~m}^{2} \mathrm{~s}^{-1}$, and the simulation step $\Delta t=1 \mathrm{~ms}$. The dashed curve is not fitting but superimposed a Gaussian distribution function calculated from Equation 7.

\section{Surface bounces the molecules back}

Then I simulate a cylindrical rod volume with various cross/end area, length $100 \mu \mathrm{m}$ (semi-infinitely long) mimicking a flow cell with $100 \mu \mathrm{m}$ cell height and filled with an aqueous solution of the molecules used in the control simulation with concentrations around 1 micromole per liter $(\mu \mathrm{M})$. All concentrations are converted to numbers of molecules per $\mathrm{m}^{3}$ volume. The average solute distance over $3 \mathrm{D}$ is $118 \mathrm{~nm}$ for 1 
$\mu \mathrm{M}$ solution and $1.2 \mu \mathrm{m}$ for $1 \mathrm{nM}$ solution but the average distance along the rod axis (1D) varies over the area of interest. The 1D diffusion movie of these molecules is recorded over time with step size $\Delta t=$ $1 \mathrm{~ms}$. Complex solute-surface interactions are ignored which has been observed in real experiments. ${ }^{41}$ All inter-solute interactions are also ignored.

The molecules randomly walk up and down the rod. Fig. 3A shows a 1D trajectory of one molecule over the condition that each time a molecule hits the ends of the rod volume, it gets mirror-bounced back into the bulk (each event is shown in the figure a red circle). The number of hitting events is summarized and compared to the predicted values (Equation 9). When a molecule hits the end wall, it has a higher chance to hit the end again later because the surface is near the maximum of the Gaussian-decay PDF of this just-bounced-back molecule. The counting events are summarized in the cumulative hitting events shown in Fig. 3B. These are all linear curves indicating a constant hitting rate proportional to the area of interest which is intuitive, i.e. the same number of molecules are observed in each frame of the movie, different than our worry that the hitting number will be curved over time.

When changing the cross area of the rod, i.e. the volume, the number of molecules hitting the end wall is changed linearly with the area. This same linear relationship is found for fixing volume and changing solute concentration. We can see at a very low number, close to one molecule in the volume when the simulation must round up the number and do not have enough hitting events to average, the simulation introduces a large noise (Fig. 3C). It is surprising that even at the low statistical region, the simulations match the predicted values in the right order of magnitude. At just $>2$ molecules level, the simulation is remarkably consistent with the prediction (Equation 9).

(A)
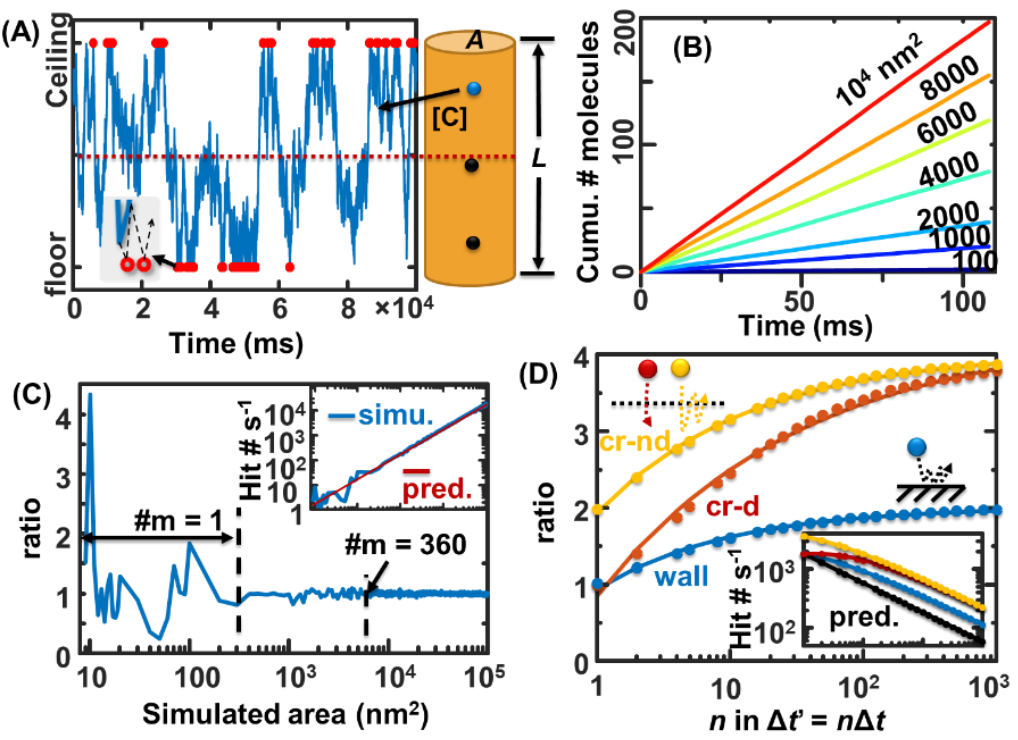

Fig. 3. (A) A randomly chosen trajectory of a molecule in a cylindrical volume (rod) where the 1D diffusion in the axel direction is simulated. The dashed line indicates an imaginary interface placed in the middle point of the bulk solution. The red circles indicate bouncing-back events happen either at the ceiling or the floor of the rod. Inset showing a case when the molecule hits the wall twice in three simulation steps. The length of the rod is $L=100 \mu \mathrm{m}$, the cross/end area of the rod varies a few orders of magnitude from $A=5$ to $10^{5} \mathrm{~nm}^{2}$, the molecular concentration in the rod volume is fixed at $C=1$ $\mu \mathrm{M}$, the simulation step time $\Delta t=1 \mathrm{~ms}$, and total simulation time $100 \mathrm{~s}$. (B) The cumulated number of molecules counted at $1 \mathrm{~ms}$ resolution over time for a different area of the rod. Linear curves are observed of all simulations. (C) The ratio of the average number of hitting events per second per simulated area to the ends of the rod walls counted at $1 \mathrm{~ms}$ resolution between the simulations and the predicted values from Equation 9 (inset showing the two curves with the same $x$-axis). The curve is divided into 
3 zones by the two dashed lines, 1, 2-360, and 360-6000 molecules in the simulated rod volume. (D) The ratio of the average number of hitting events vs the number of bins of counting cycles between the simulations and the predicted values from Equation 9 using $\Delta t$ ' (inset showing the curves with the same $x$-axis but with $y$-axis number of hit event per second per simulated area). Simulated with rod area $10^{4} \mathrm{~nm}^{2}$ (602 molecules in this volume), step time $1 \mathrm{~ms}$, and total simulation time $100 \mathrm{~s}$. The curve labeled 'wall' represents the average hitting to the two ends of the rod (fitted $y=(2.0 \pm 0.1)-$ $\left.(1.0 \pm 0.1) x^{-(0.44 \pm 0.03)}\right)$; curve 'cr-d' represents the molecules crossing the imaginary interface in one direction (averaged of the two directions, fitted $\left.y=(4.3 \pm 0.1)-(3.5 \pm 0.2) x^{-(0.28 \pm 0.01)}\right)$; curve 'crnd' represents the sum of the molecules crossing the imaginary interface from both directions (fitted $\left.y=(4.0 \pm 0.1)-(2.1 \pm 0.1) x^{-(0.38 \pm 0.02)}\right)$.

We already have the clue to answer the non-intuitive question of the measuring-time-depending hitting rate from Fig. 3A. Because the same molecule bouncing the same surface multiple times during a single measuring time is only counted once in our Gaussian PDF model, longer time-resolution will give a smaller hitting rate and vice versa, i.e. the drop of the number with increasing integration time is because the fine collisions of the same molecule are grouped in each measuring cycle. The hitting number vs time curves will all be linear with different slopes if we change the integration time. A set of curves with one measuring time and different areas are shown in Fig. 3B.

Reducing the counting/measuring time resolution can be simulated by binning the same trajectory into longer step times and forcing the maximum number of hits per frame for each molecule to one. Fig. 3D confirms that the number of hitting events per second indeed drops as the decrease of the counting frequency as predicted by Equation 9 (Fig. 3D inset).

We also get an answer from Fig. 3D for the factor of two disagreements between the first term of the Ward-Tordai equation (Equation 5) and Equation 9: correction for the fractal nature of diffusion should be applied to our single-molecule method. The simulated value for collision number to the wall in a frame approaches two times the predicted values (Equation 9) at $\sim 1000$ times longer than the simulation step time for the bouncing surface (Fig. 3D). Since the $1 \mathrm{~ms}$ time resolution is arbitrarily chosen to represent the net effect of much finer diffusion steps (ultrafast dynamics), we can argue that if we had simulated the diffusion at $<1 \mu$ s resolution, Equation 9 would be corrected 2 times to describe the $1 \mathrm{~ms}$ simulation to a closer format to the Langmuir-Schaefer equation:

$$
\Gamma(\text { in } \Delta t)=2 A C \sqrt{\frac{D \Delta t}{\pi}}
$$

except for $\Delta t$ is used instead of $t$. If we had increased the time resolution to picosecond (ps), The RMSD in the solution would have been down to sub-water size e.g. water rotating $\sim 1.8$ ps. ${ }^{42}$ Ultrafast collision is not the interest of my simulations where the diffusion constant is described by the Langevin equation. ${ }^{1}$ We can hypothesize for now that at ultrafast time resolution, the large number of collisions per second predicted by Equation 11 (if still holds) divided by $\Delta t$ is dominated by the recurrent collisions of the same molecules to the surface when they approach each other. Relatively long waiting time may need to see another molecule to come (averaged to a small value of collision predicted by Equation 11). If the Gaussian diffusion argument holds on all time scale, i.e. a fractal equation that holds self-similarity over all time scales, the actual hitting number and rate for all time steps above nanosecond should converge to two times the values predicted by Equation 9 and 10. These two equations are still used as the reference equations in some of the rest simulations which represents the limit of information for the simulations near its step resolution. 
11 to be

The average rate of solute colliding the wall measured in $\Delta t$ is then corrected from Equations 9-

$$
\langle r\rangle_{(\text {meausred in } \Delta t)}=2 A C \sqrt{\frac{D}{\pi \Delta t}}
$$

It is surprising at first and then reasonable to me that the mirror effect is not observed for the hitting frequency to the solid walls but rather observed for the imaginary interface placed in the bulk solution (Fig. 3D). As a comparison to the two-fold correction for the solid walls (Equation 11), the frequency of molecules crossing an imaginary interface place in the middle of the simulation space approaches four times the predicted values from one side to another and also four times from both sides (Fig. 3D). When the simulations don't contain the fractal information, the number of molecules crossing from one side to another side of the imaginary interface each time frame is precisely predicted by Equation 9 for the one-step simulations (not binned data). The crossing rate of the molecules from both sides is twice as expected for the same data. However, when binned (containing the fractal information), both approaching four times the value of Equation 9. This doubling at the imaginary interface than the solid interface can be explained by the mirror effect. The PDF at the imaginary interface is doubled because the probability function after the molecule has passed the interface mirrors the original probability function. It is obvious that when removing the directional restriction, the molecule crosses the interface two times probable than from one side to the other because it is equally probable for another molecule on the other side to pass the interface at the shorter time interval. However, when the measuring time is longer, it is interesting to find that the one-side crossing approaches to the both-side crossing frequency because the rate-limiting step becomes the number of the molecules near the interface which is the same for both cases, i.e., the molecule will go up and down the imaginary interface many times no matter which the final direction of crossing it takes at the end of a measuring time frame. Adapt the same fractal argument for the hitting rate to the wall, the one-step simulation on the imaginary interface in the bulk solution lower estimated the rate and should be corrected by a fact of four. This correction is an important conclusion for analyzing molecular interactions in the bulk solutions.

To summarize the above analysis, the linear curves in Fig. 3B have a special restriction, each molecule if hitting the surface is only counted once in the counting cycle/frame with the integration time of $1 \mathrm{~ms}$. The slopes of these curves are predicted by Equation 10 with $\Delta t=1 \mathrm{~ms}$. Changing the counting frequency will change the slope which either unfolds or folds the fine collision frequencies of the same molecule during each integration time but the linear relationship maintains, i.e. intuitively each frame has the same number of collisions. The dependence of the slope with the counting time is a nonlinear curve predicted by Equation 10, i.e., non-intuitively the number gets only the square root of two increased when double the integration time. The effect of fractal diffusion and the mirror effect at an imaginary interface is only observable for a simulation that has enough hidden information among each step to simulate the fractal nature of the diffusion, i.e. in binned diffusion simulations. The simulations ignore such effect (non-binned data) only reflect the hitting rate predicted by Equation 10 for both cases (Fig. 3D the first points of the curves).

To correct the slope of the collision number predicted by the simulations without the fractal information, a factor-of-two correction to Equation 10 is needed (Equation 11) for molecules to a solid wall; and a factor-of-four correction is needed for the collisions in the bulk solution.

Equation 11 is consistent with the experimental measurements we have reported before. ${ }^{34}$ When $5 \mathrm{nM}\left(C=3 \times 10^{18} \mathrm{~m}^{-3}\right)$ YOYO-1 dye molecule in water with $D=2.9 \times 10^{-10} \mathrm{~m}^{2} / \mathrm{s}$ is adsorbed to a $1-\mu \mathrm{m}-$ long DNA molecule with an area $\sim 2000 \mathrm{~nm}^{2}$ immobilized on a surface, the frequency measured at $50 \mathrm{~ms}$ time resolution is $0.4 \mathrm{~s}^{-1}$. Equation 11 predicts a rate of $0.5 \mathrm{~s}^{-1}$, indicating $50 \mathrm{~ms}$ is a proper time resolution to calculate the actual binding rate. It also indicates that we can use the Langmuir-Schaefer equation to precisely measure the diffusion constant of solute molecules in the solution. 
Equation 11 is still very different from Equation 5 because our model predicts curves with a linearly increased number of collisions over time for a non-adsorptive bouncing surface with fixed $\Delta t$, i.e., as a constant in the equation representing the measuring time resolution while the Langmuir-Schaefer equation uses the actual time. We will search for an answer to this question in the next simulations (mind experiments).

\section{The surface no matter if empty or occupied absorbs the molecules upon collisions}

Assume a case that the diffusing probe absorbs on the surface when they hit (for high-affinity binding such as electrostatic binding, antibody-antigen interactions, and surfactants adsorbing to air-water interface in the original Ward-Tordai experiments), the rebounding events discussed above for a bouncing surface vanishes, and the concentration of the solutes near the surface drops creating an evolving concentration gradient. ${ }^{29}$ It is interesting to simulate the adsorption kinetics of the molecules under this condition. Such adsorptions have been described by the Langmuir adsorption model which begins with a diffusionlimited region and projects to a surface-area-limited region. To begin with, we assume that the surface will adsorb the molecule whenever it hits the surface, i.e., during the short-time limit when reducing of active surface area due to adsorption is negligible. I artificially extend the time to a longer period during this mind experiment to simulate the effects of the adsorption to the sub-surface (bulk). Several rods with the same length and different cross areas are simulated to check the effect of the artificial discrete distance among the molecules along the rod axis during the simulations.

Fig. 4 shows the results of the simulations of adsorption of solute to the floor of a rod when the ceiling is non-adsorptive. The surface-area limitation is ignored in this simulation, i.e., the surface can take an infinite amount of molecules. This is just a mind experiment for now. A more realistic surfacelimited experiment will be simulated later. The accumulated number of molecules adsorbed goes up faster in the beginning and slow down later (Fig. 4A). The adsorption rate, $\langle r(t)\rangle=\frac{\Gamma(t+\Delta t)-\Gamma(t)}{\Delta t}$, where $\Gamma$ is the accumulated number of molecules adsorbed in the area, decays over time (Fig. 4B). When normalized to the predicted value from Equation 9, no significant area dependence is observed among the simulation areas (Fig. 4B). The smaller the rod area, the fewer molecules, and the larger the noise level. The decay of the adsorption rate over time is consistent with the evolving degree of concentration gradient near the surface (Fig. 4C), confirming an important assumption of Langmuir-Schaefer adsorption isotherm. At time 0 when there is no concentration gradient, the adsorption rates are the same as the predicted value. The gradient has the consistent exponential-like shape as Ward and Todai has hypothesized. ${ }^{25}$ The rate quickly drops to $\sim 10 \%$ of the predicted value at $100 \mathrm{~ms}$, and $\sim 1 \%$ at $5 \mathrm{~s}$. At $5 \mathrm{~s}$, the concentration gradient has projected to the whole rod volume, when the concentration at the ceiling has dropped below the initial concentration of $1 \mu \mathrm{M}$ (represented by the histogram bar height 6 and bar width $1 \mu \mathrm{m}$ ).

All the curves of the cumulated number of molecules in Fig. 4A and $4 \mathbf{E}$ can be fitted with an equation similar to Equation 9 and Equation 11 and are independent on the measuring time resolution $\Delta t$ :

$$
\Gamma(t)=\alpha A C \sqrt{\frac{\beta D t}{\pi}}
$$

where $t$ is the actual time from the beginning of the adsorption instead of the counting time resolution $\Delta t$, $\alpha$ and $\beta$ are fitting constants with $\alpha$ within 1.7-2.0 and $\beta$ within 0.9-1.0. When the slope (derivative) of Equation 13 is normalized with the initial rate at $t=\Delta t=0.001 \mathrm{~s}$, a trendline of $r(\mathrm{t})=0.03 t^{-0.45}$ is observed (Fig. 4E), meaning, for the simulated mind experiment, the rate will drop to $3 \%$ of its initial rate at $1 \mathrm{~s}$ because of the concentration gradient near the surface evolved (when the surface-area limitation is ignored). 

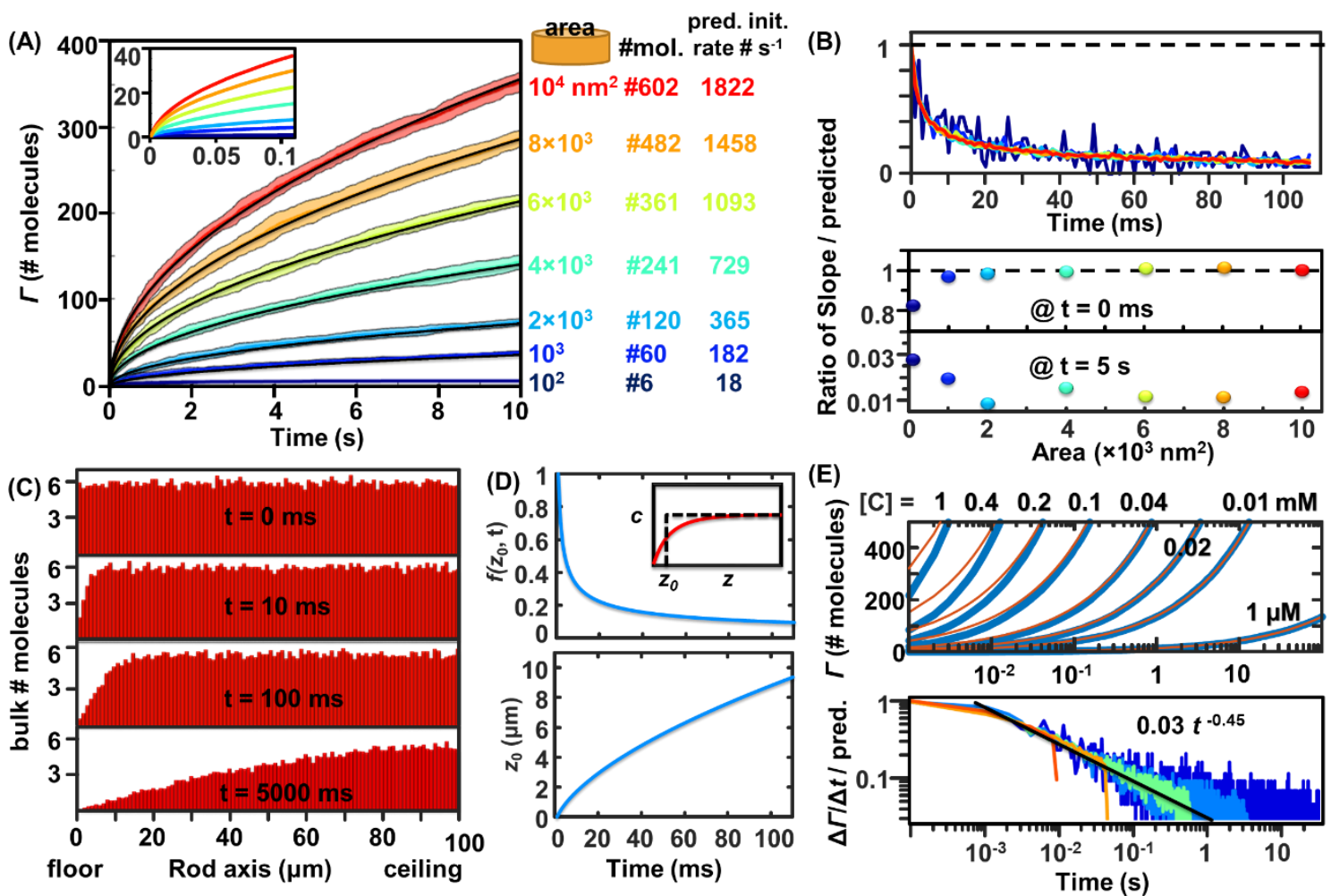

Fig. 4. (A) Accumulated adsorption curves of molecules 1D diffusing in a rod volume, bouncing on the ceiling, and adsorbed on the floor at each hitting event. The molecules in the rod are depleted over time with no replenishment. Only the 1D diffusion is simulated. The length of the rod is fixed at $100 \mu \mathrm{m}$. The concentration of the molecule is $[C]=1 \mu \mathrm{M}$ with $D=2.88 \times 10^{-10} \mathrm{~m}^{2} / \mathrm{s}$. The step time is $\Delta t=1 \mathrm{~ms}$. The cross-area of the rod, the initial total number of molecules, and the initial rate (first frame) are labeled. The shadows indicate the standard deviation of 10 simulations. The black curves are fittings using Equation 13. Insets show the average of 1000 simulations of the first $0.1 \mathrm{~s}$. (B) The slope of the curves in (A) normalized to the predicted initial rates with two snapshots shown. (C) The average histograms of the molecule locations along the rod axis of 100 simulations with rod area $10^{4} \mathrm{~nm}^{2}$ at a few selected snapshot time. (D) A correlation was obtained using Equation 16 as an approximation to a virtual gap model (inset) for the gradients in (C). (E) Adsorption curve on the floor of a rod with length $1 \mathrm{~mm}$ and cross-area $1000 \mathrm{~nm}^{2}$, and various solute concentrations as labeled. The thickness of the blue curves represents the standard deviations of 20 simulations. The initial curves are all consistent with the predicted values of Equation 9, but the whole curve is better fitted with Equation 13 (red curves) than Equation 9. The decay of the slope, when normalized to its initial slope, follows a power-law decay trendline $0.03 t^{-0.45}$, where time $t$ is in unit s.

Equation 13 is identical to the Langmuir-Schaefer equation, i.e., the first term of the Ward-Tordai equation (Equation 5) when taking $\alpha=2$ and $\beta=1$ within the simulation error. Interestingly, the fractal nature of the diffusion is less a problem in this case than the bouncing surface. One adsorption in each simulated step may have been already satisfied in this case, or another argument is that the rate-limiting step is transporting the molecule to a place near the surface.

What is the effect of measuring frequency during this short-time-limit stage? According to Equation 13 which is independent of the measuring frequency, I conclude that the counting frequency does not affect the binding curves of the adsorption which are determined independently on how one measure, but the effective initial rate constants measured discretely is power-low dependent on the counting frequency, 
i.e. dependent of the concentration gradient near the surface which is a function of time because the concentration gradient evolving over time makes the rate non-linear vs time. This conclusion is consistent with the time-dependent fractal reaction rate constant in diluted solutions. ${ }^{39}$ In the above simulation, $\Delta t=$ $1 \mathrm{~ms}$ is adapted as the reference frequency. We are going to find the relationship between the slopes of the accumulated number of molecules at time $t$ (i.e. rate) and its initial (first frame) measured rate with integration time $\Delta t$. $=1)$ :

The true rate at time $t$ (transient slope of) is the first derivative of Equation 13 (let $\alpha=2$ and $\beta$

$$
r(t)=A C \sqrt{\frac{D}{\pi t}}
$$

Equation 14 suggests that the linear increase of the number of collisions for a bouncing surface no longer holds but rather a power-law decay is observed for the adsorptive surface. When using the initial rate counted at the first measuring cycle $\Delta t$ as the reference, and approximating the gradual concentration gradient as a sudden turning on curve starting at $z_{0}$ from the surface (Fig. 4D inset), Equation 14 can be rewritten as

$$
r(t)=A C \sqrt{\frac{D}{\pi t}}=\int_{z=z_{0}}^{\infty} \frac{C A}{\Delta t} \mathrm{~d} z \int_{x=z}^{\infty} \frac{1}{\sqrt{4 \pi D \Delta t}} e^{-\frac{x^{2}}{4 D \Delta t} \mathrm{~d} x}=f\left(z_{0}, t\right) A C \sqrt{\frac{D}{\pi \Delta t}} \quad(t>=\Delta t)
$$

With $\Delta t=1 \mathrm{~ms}, D=2.88 \times 10^{-10} \mathrm{~m}^{2} \mathrm{~s}^{-1}, C=1 \mu \mathrm{M}$, and $A=10^{4} \mathrm{~nm}^{2}$, we can see that within the "shorttime limit", $f\left(z_{0}, t\right)=\sqrt{\frac{\Delta t}{t}}$, which equals 1.0, 0.3, 0.1, and 0.014 for $t=1 \mathrm{~ms}, 10 \mathrm{~ms}, 100 \mathrm{~ms}$ and $5 \mathrm{~s}$ slope respectively (Fig. 4B, 4C). The effective gap $z_{0}$ solved numerically correspond to values $0 \mathrm{~nm}$, $610 \mathrm{~nm}, 1.0 \mu \mathrm{m}, 2.3 \mu \mathrm{m}$ respectively.

The value of $z_{0}$ can also be solved analytically, such that

$$
f\left(z_{0}, t\right)=\sqrt{\frac{\Delta t}{t}}=1-\operatorname{erfc}\left(\frac{z_{0}}{\sqrt{2 D t}}\right)=1-\frac{2}{\sqrt{\pi}} \int_{0}^{\frac{z_{0}}{\sqrt{2 D t}}} e^{-\tau^{2}} d \tau \quad(t>=\Delta t)
$$

where $\tau$ is a dummy variable (Fig. 4D). Thus, the fraction is related to the error function (erfc) of the Gaussian PDF that spreads out over time. Equation 16 is consistent with the numerical solutions of

\section{Equation 15.}

Now it is confusing what $\Delta t$ should be chosen to calculate the "initial adsorption rate". During the first measuring cycle, time $0-\Delta t$, do we consider an evolving sub-surface-bulk concentration gradient or do we consider a uniform concentration across the bulk as time 0 ?

We can do a mind experiment with molecules aligned perfectly in space shown in Fig. 5. To maintain the same molecular distribution within the time $\Delta t$, the average location of the molecule should be the same as the distance between two molecules, i.e. the net effect is they just switch positions during this time:

$$
d_{0}=\frac{1}{\sqrt[3]{C}}=\frac{\int_{0}^{\infty} z e^{\frac{-z^{2}}{4 D \Delta t}} d z}{\int_{0}^{\infty} e^{\frac{-z^{2}}{4 D \Delta t}} d z}=\sqrt{\frac{4 D \Delta t}{\pi}}
$$

where $d_{0}$ and $z$ are shown in Fig. 5. Thus, the characteristic integration time $\Delta t_{\mathrm{c}}$ to calculate the average adsorption rate with no sub-surface concentration gradient is: 


$$
\Delta t_{c}=\frac{\pi}{4 D C^{2 / 3}}
$$

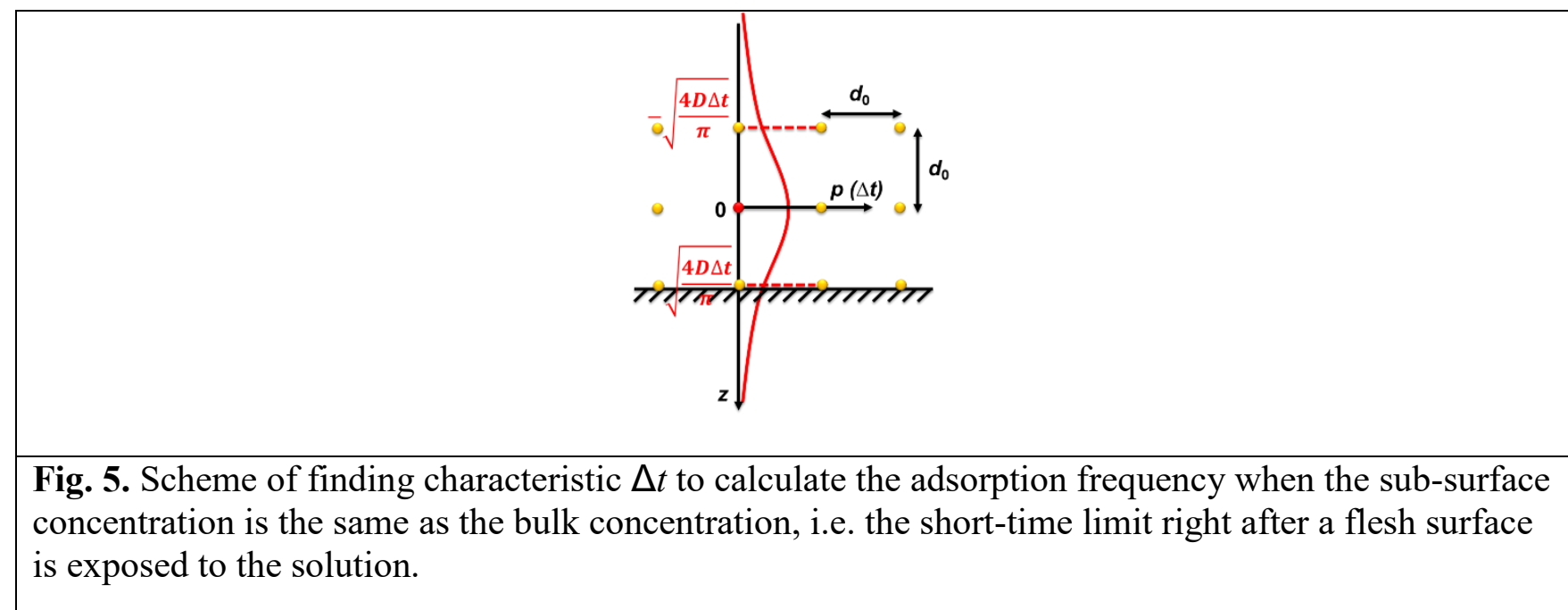

Thus, the average adsorption rate can be calculated by Equation 12:

$$
\langle r\rangle=2 A C \sqrt{\frac{D}{\pi \Delta t_{c}}}=4 \pi^{-1} A C^{4 / 3} D
$$

This equation has the correct unit $\mathrm{s}^{-1}$ for the dimensional analysis and is consistent with another calculation assuming the molecular exchanging time $\Delta t_{\mathrm{c}}$ is the average adsorption time for the characteristic surface area $d_{0}^{2}$. That is

$$
\langle r\rangle=\frac{A}{d_{0}^{2}} \frac{1}{\Delta t_{c}}=4 \pi^{-1} A C^{4 / 3} D
$$

In short, $\Delta t_{\mathrm{c}}$ is the characteristic diffusion-limit time for the adsorption. Shorter than this time, the high average hitting rate and the low total number of adsorptions predicted by Equation 13 and 12 are due to the isolated but repetitive collision of the same molecules on the surface. Longer than this time, the same molecule might have diffused away beyond the first nearby layer and diffuse back to the surface which should have been counted as different molecules if the sub-surface concentration has been maintained, i.e., Equation 13 has lower estimated the collision frequency longer than this time. Any subsurface concentration gradient if established after this time will induce the drop of collision rate (transient) over time shown by Equation 14. If the concentration gradient is refilled faster than this time, the rate of adsorption will maintain over time with the value predicted by Equation 19. Thus, at the very beginning of the adsorption, $\Delta t_{\mathrm{c}}$ is the time the molecules have just collided with the surface and their first nearest neighbor in the subsurface zone is about to collide the surface.

\section{The surface only when empty absorbs the molecules upon collisions}

Now we are just missing the back-diffusing term of Equation 5. Note that the above mind experiment is for adsorption with no activation energy barrier consistent with the original Ward and Tordai model, ${ }^{25}$ and no surface rejection due to pre-occupation which is different than they have pictured. The back-diffusion term, as the name suggests, rises when the surface started to be covered by the molecule 
and gains the ability to reject molecules (which then diffuse back to the bulk) such that the active surface area is non-linearly decreased. ${ }^{29}$ When there is an energy barrier, the surface will also reject a fixed potion of the molecules which will make the concentration gradient less intensive and the drop of the speed less steep over time. When the adsorption approaches the surface-area-limited region (e.g., of a Langmuir adsorption), the sub-surface concentration gradient recovers due to the decrease of the adsorption rate. Thus, when the rejection happens, it changes the concentration gradient dynamics in the subsurface making the Ward-Tordai equation very complicated, which predicts that the overall effect makes the adsorption slower than the value the Langmuir-Schaefer equation (Equation 13) predicts.

In the original Ward-Tordai paper, ${ }^{25}$ the back-diffusion term is experimentally determined by measuring the adsorption rate and back-calculate the sub-surface concentration. From the above discussion, we can see that our Monte Carlo simulation can predict the whole adsorption with the back-diffusion curve if we add a surface reaction model, e.g. the Langmuir adsorption model. With a moderate size of simulation (1000 molecules, time step $1 \mathrm{~ms}$, total time $100 \mathrm{~s}$ ), we can simulate the adsorption curve containing the back-diffusion term in $\sim 5$ seconds with a regular desktop computer, making it possible to fit experimental data with a method we have recently adapted. For example, we have fitted kinetic data using our home-made code jcfit (Github) to optimizing simulations for a given set of data. ${ }^{40,43}$

For the back-diffusion term, I am curious about simulating a routing experiment I have done before, self-assembly of alkanethiol molecules on gold or $\mathrm{ZnO}$ surface, ${ }^{44}$ particularly in how long it takes to reach a whole surface coverage with no convection and stirring. Thus I simulate the adsorption kinetics of octadecanethiol in ethanol (concentration from $10 \mu \mathrm{M}$ to $1 \mathrm{mM}$ ) on a flat surface assuming the adsorption probability in binary using the Metropolis Monte Carlo Method. ${ }^{45}$ Specifically, molecules adsorb on an empty area and bounce on pre-occupied area upon hitting, i.e., $p_{\text {ads }}=1-\theta$ for each hitting event, where $\theta$ is the surface coverage normalized to 1 at full coverage and surface seats are set to 4 molecules per square nanometer. ${ }^{44}$ No molecules desorbed from the surface are allowed during the simulation. These simulations are Langmuir adsorption processes with zero activation energy barriers as Ward and Tordai have assumed. In the future, if needed, an activation energy barrier $E_{\mathrm{a}}$ can be introduced to reduce the adsorption probability $\exp \left(-E_{\mathrm{a}} /(R T)\right)$ and a desorption rate proportional to the surface coverage can be introduced for data fitting and mechanistic study of surface adsorption models.
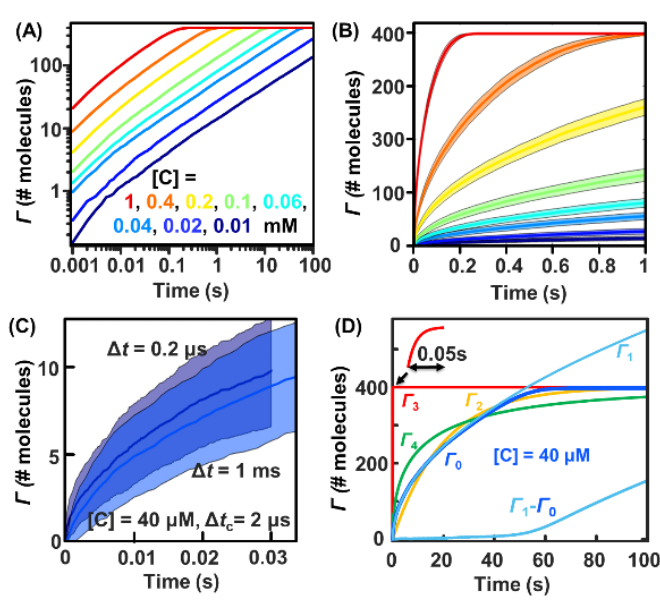

Fig. 6. (A) Simulated adsorption curve of octadecanethiol/ethanol solution on an adsorptive surface. Curves are averaged from 100 simulations. Diffusion along the rod axis (1D) is simulated with rod length $1 \mathrm{~mm}$, cross-area $100 \mathrm{~nm}^{2}$ (assuming 400 molecules adsorbed at full coverage $\theta=1$ ), diffusion constant $D=4.13 \times 10^{-10} \mathrm{~m}^{2} \mathrm{~s}^{-1}$, time step resolution $\Delta t=1 \mathrm{~ms}$ and various solution concentrations as color-coded and ordered. (B) Zoom in of (A) the first $1 \mathrm{~s}$ data. The simulated curves are color-coded with the shadows being the standard deviations of the 100 simulations. (C) Comparing $\Gamma$ for $[C]=0.04$ $\mathrm{mM}$ simulated at $0.2 \mu \mathrm{s}$ time resolution and $1 \mathrm{~ms}$ time resolution. Shadow is the standard deviation of 
100 simulations. (D) Overlay of a simulated example curve $\left(\Gamma_{0}\right.$, blue, $\left.[C]=0.04 \mathrm{mM}\right)$ with different models explained in the text. The curve " $\Gamma_{1}-\Gamma_{0}$ " is the subtraction of the Langmuir-Schaefer equation $\left(\Gamma_{1}\right)$ with the simulated data $\left(\Gamma_{0}\right)$ which represents the back-diffusion term of this adsorption curve for the Ward-Tordai equation.

The curves containing the simulated back-diffusion term for Langmuir isotherm is shown in Fig. 6A and 6B to represent the "experimental data". The similarity among the curves simulated with different solute concentrations (Fig. 6A) suggests that they share the same diffusion-controlled mechanism. The value of $\Delta t_{\mathrm{c}}$ is in $\mu \mathrm{s}$ for the concentrations simulated, e.g., $6 \mu \mathrm{s}$ for the $10 \mu \mathrm{M}$ solution, and $2 \mu \mathrm{s}$ for the $40 \mu \mathrm{M}$ solution. Comparing with the simulations done with faster time resolution than $\Delta t_{\mathrm{c}}(\mathbf{F i g}$. 6C), the effect of the longer-than- $\Delta t_{\mathrm{c}}$ simulation time cycle $\Delta t=1 \mathrm{~ms}$ is as expected concentrated in the first $1 \mathrm{~ms}$ which shifts the curve but has little effect on the overall shape and magnitude of the curve. Thus, we assume the curves simulated at $1 \mathrm{~ms}$ time resolution represent the Langmuir adsorptions for ideal systems with no stirring. We are going to analyze these curves with a few different models.

First, the Langmuir-Schaefer equation is copied here for ease of reading (Fig. 6D):

$$
\Gamma_{1}(t)=2 A C \sqrt{\frac{D t}{\pi}}
$$

This non-surface-area-limited adsorption model is wildly used in the literature for the short-time approximation when the ideal sub-surface concentration gradient is established (consistent with the simulations in Fig. 6).

When we consider the surface-area limitation, the Langmuir single-molecule adsorption reaction model can be adopted (which is the model we simulated but assuming hidden from the analysis). Assume the first-order reaction between the empty surface $(\mathrm{S})$ in unit number of open seats for molecules and the number of adsorbed molecules $\mathrm{S}^{*}$ :

$$
S \stackrel{k}{\rightarrow} S^{*}
$$

Thus,

$$
\frac{d[S]_{t}}{d t}=-k[S]_{t}
$$

where $[\mathrm{S}]_{t}$ is the number of empty seats available for new molecules to adsorb and $k$ is the time-dependent rate constant for the Ward-Tordai equation.

Second, if we assume an effective rate constant $k_{2}$ to represent the average rate constant of the whole adsorption process (Fig. 6D),

$$
\Gamma_{2}(t)=\left[S^{*}\right]_{t}=[S]_{0}\left(1-e^{-k_{2} t}\right)=\frac{A}{a}\left(1-e^{-k_{2} t}\right)
$$

where $\left[\mathrm{S}^{*}\right]_{\mathrm{t}}$ is the number of molecules adsorbed at time $t$, and $[\mathrm{S}]_{0}=A / a=400$ is the total number of empty seats on the surface at time 0 . Thus, $k_{2}$ can be fitted from the "experimental data" to represent the average of the Ward-Tordai equation in the Langmuir adsorption model. ${ }^{28}$

Third, now let us assume an extreme condition to be the upper limit of the rate constant, that the surface concentration gradient is negligible and compare such condition with the simulations, i.e. an ideal condition suitable to approximate the early stage of systems with high bulk concentration, and/or 
good stirring/flow, or a small area on the surface surrounded by a bouncing surface (the last being a typical biosensing system). The rate constant is predicted to correlate with the characteristic integration time $\Delta t_{\mathrm{c}}$ by Equation 19 to be (Fig. 6D):

$$
\begin{aligned}
& k_{3}=4 \pi^{-1} a C^{4 / 3} D \\
& \Gamma_{3}(t)=\frac{A}{a}\left(1-e^{-k_{3} t}\right)
\end{aligned}
$$

Fourth, the time-dependent back-diffusion term of the Ward-Tordai equation (Equation 5) can be solved by subtracting the data with the Langmuir-Schaefer equation (Fig. 6D, the $\Gamma_{1}-\Gamma_{0}$ curve). Let's just approximate the rate of the whole Ward-Torddai equation with an average correction value $\left\langle w d>_{\mathrm{C}}\right.$. Then Equation 5 generates (Fig. 6D),

$$
\begin{aligned}
& k_{4}=\langle w d\rangle_{C} a C \sqrt{\frac{D}{\pi t}} \\
& \Gamma_{4}(t)=\frac{A}{a}\left(1-e^{-2 k_{4} t}\right)
\end{aligned}
$$

where $A$ is the total area $\left(\mathrm{m}^{2}\right), a$ is the area each molecule will occupy after adsorption $\left(\mathrm{m}^{2}\right), C$ is the number concentration $\left(\# \mathrm{~m}^{-3}\right), D$ is the diffusion constant $\left(\mathrm{m}^{2} \mathrm{~s}^{-1}\right)$, and $t$ is time (s).

We can now compare the four different models (Fig. 6D). The Langmuir-Schaefer equation $\left(\Gamma_{1}\right)$ is the best model for the simulated Langmuir adsorption curve up to $\sim 80 \%$ of the saturation coverage. But it over-saturates the surface indicated by the fast turning up of the back-diffusion curve $\left(\Gamma_{1}-\Gamma_{0}\right)$ after. The fitted curve $\left(\Gamma_{2}\right)$ represents the overall shape of the adsorption curve the best among the four models but lower-estimate the initial slope the most causing trouble for models rely on this part of data. The higher the concentration, the better this model works (fitting $\mathrm{R}^{2} \sim 1$ for $1 \mathrm{mM}$ curve and $\sim 0.9$ for $10 \mu \mathrm{M}$ curve). The ideal-stirring curve $\left(\Gamma_{3}\right)$ represents the theoretical upper limit of the adsorption rate which predicts saturation in the millisecond region (Fig. 6D inset). Because the measuring time resolution $(\Delta t=1 \mathrm{~ms})$ is longer than the characteristic diffusion time $\left(\Delta t_{\mathrm{c}}\right)$, the initial slope of the first cycle of $\Gamma_{1}$ lower-estimates the maximum initial rate that is represented by the initial slope of $\Gamma_{3}$. The averaged Ward-Tordai equation $\left(\Gamma_{4}\right)$ catches the initial slope of $\Gamma_{3}$ and the far end after the saturation but performs poorly in between. The value $\langle w d\rangle_{\mathrm{C}}$ is fitted to be between 1 and 2 for the several concentrations simulated. Comparing the effective rate constant $k_{2}$ with the maximum (initial) rate constant $k_{3}, \sim 3$ orders of magnitude difference is observed between these to models.

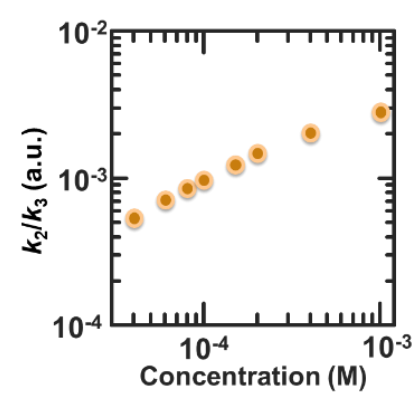

Fig. 7. Comparison of $k_{2}$ (effective rate constant from $\Gamma_{2}$ ) and $k_{3}$ (theoretical maximum from $\Gamma_{3}$ ) for the simulations in Fig. 6. 
The simulation results in Fig. 6 are consistent with the experimental results in the literature. In the simulation, at millimolar solute concentrations, the surface reaches the full coverage within a second. At the micromolar level, it takes over $100 \mathrm{~s}$ to saturate the surface. ${ }^{46}$ With convection, flow, or mild stirring in real experiments, the adsorption rate is faster than the values of the simulations. Typical thiol on gold experiments is carried out at a longer time though. It is a slower process than adsorption to convert physical adsorption to chemical adsorption, maximize the surface coverage via rearrangement of the thiols on gold, and minimize the number of defects. ${ }^{47}$

A real experiment of adsorptions with no energy barrier will generate a curve that lies between the ideal-stirring curve $\Gamma_{3}$ and the purely diffusion-controlled simulated curve $\Gamma_{0}$ (Fig. 6D space between the red and the blue curves). The curve $\Gamma_{3}$ represents the theoretically fastest adsorption of the given condition. The simulated curve represents the slowest reaction with no convection and no stirring. When there is an adsorption energy barrier, the adsorption curves my end up on the right side of the simulated curve, i.e. slower than $\Gamma_{0}$.

The uncertainty in the fluidic condition during the real experiments makes the Ward-Tordai equation not suitable to measure the diffusion constants of the solutes which often introduces 5-15 orders of magnitude of difference ${ }^{25,29}$ than the values predicted by the Einstein-Stoke equation or the values measured by other more direct techniques such as single-particle tracking. It also has limited the applications of using the Ward-Tordai equation to predict adsorption rate from the bottom-up with the first principle of diffusion. However, with the power of modern simulations, it may become a necessary and powerful equation to predict diffusion problems in chemistry, biochemistry, chemical engineering, and many other fields such as the border problem of defending infectious disease during a pandemic.

\section{SUMMARY}

In summary, the ensemble solution of adsorption kinetics namely the Langmuir-Schaefer equation and the Ward-Tordai equation (Equation 5) have been reproduced using single-molecule diffusion probability density function with clarification for the effects of measuring time resolution and corrections for the fractal nature of diffusion (Equation 13). The most unique conclusion drawn from the single-molecule approach is that there is a characteristic integration time $\Delta t_{\mathrm{c}}$ for the equations which has never been a consideration for the ensemble approaches, a missing piece of the Ward-Tordai equation. That is, the characteristic time distinguishes the over-estimated multiple collisions from the same molecule shorter than this time and the lower-estimation of the collisions longer than this time. The correlation between the ensemble solution and the single-molecule solution also suggests careful corrections and treatment should be applied to the results of any single-molecule Monte Carlo simulation targeting solving diffusion problems.

We can draw some interesting specific conclusions from the combination of the results from the theoretical analysis and the Monte Carlo simulations. When we measure the collision events of probe molecules in the bulk solution to a small target area on a flat surface, the frequency of seeing such events is depending on the measuring time resolution and how the surface reacts with the probes.

(1) If the surface just simply bounces the probe back to the bulk solution, the number of events observed per measuring cycle is dependent on the length of the time step described by Equation 11 which is corrected from the analytical solution Equation 9 after simulations. The events are stochastic, but the average number of events is the same for each cycle with a large enough observation area, i.e. the accumulated events $\Gamma(\mathrm{t})$ is a linear curve whose slope varies with the length of the time step. The simulation results suggest that this changing of the rate on time step $(\Delta t)$ is not because more molecules are visiting the surface at a shorter period but rather when boost up the observation power, the finer structure of 
diffusion is revealed to the observer. That is, when a molecule approaches the surface, it may collide with the surface many times before leaving the surface far enough and escaping into the bulk. Reducing time resolution merges these fine collisions and the collision frequency drop with a power-law decay function described in Equation 11. This equation predicts an adsorption rate of a dye to a DNA molecule that is consistent with our previous experimental measurement.

(2) If the whole surface is adsorptive and the probes stick at their first collisions to the surface, Equation 11 has to be replaced by the Langmuir-Schaefer equation (Equation 13). Equation 13 is literary the same as Equation 11 by just replacing the length of the step time $(\Delta t)$ with a real projection time $(t)$ which is independent of the observation step time resolution. This makes sense because the analytical solution (Equation 9) already considered all molecules in the bulk. Equation 13 suggests that for such an adsorptive surface, the probes bind to the surface faster at the beginning and slow down over time following the power-law decay function. The results of the simulations confirm the literature assumption that this decay of binding rate is because, over time, a concentration gradient evolves near the surface which depletes the number of available solute molecules near the surface. For large solute concentration, the surface will saturate before significant gradient forms thus the adsorption follows the first-order reaction model better. For small probe concentration, a significant gradient evolves, and the adsorption will be diffusion-controlled and follows the power-law model.

(3) It is possible to use the corrected single-molecule equation for molecules crossing an imaginary interface to predict the collision frequency of two solute molecules in the bulk solution with a further correction on the relative diffusion constant. The major obstacle for the ensemble approach, the missing characteristic diffusion time, has been revealed by the single-molecule approach in this report. In the future, this could provide more insight into the steric factor in the collision theory and extend the theory to predict reaction kinetics in diluted solutions.

(4) For single-molecule simulations to correctly reflect the fractal nature of diffusion during collision estimation, the unit step should be a few orders of magnitude faster than the targeting time resolution. This is bad news and a significant extra cost to future simulations on diffusion. A lower-cost approach might be to correct the low-time-resolution simulation results with a correction factor between 1 and 4.

From the simulated self-assembled monolayer example, we have compared the LangmuirSchaefer equation and Ward-Tordai equation under idealized conditions and have a peek of the limitation of these equations in real experiments that are much more complicated than under the ideal conditions. With this critical knowledge, we may start to applicate these simple equations and to use them carefully in various fields such as calculating the collision frequency of molecules in a diluted gas or liquid solution, membrane penetration, self-assembly, and biosensing. The single-molecule approach also makes integration of the diffusion equation for molecules in confined space colliding structured and curved surface mathematically straightforward, and thus easier to understand than the ensemble equations of diffusion. It may also find applications in finer simulations such as molecular dynamics simulations to skip noninterested mass transportation steps among the solvent.

\section{ASSOCIATED CONTENT}

Supporting Information. Sample MATLAB source codes for $1 \mathrm{D}$ and $3 \mathrm{D}$ control simulations.

\section{AUTHOR INFORMATION}




\section{Corresponding Author}

*Jixin Chen at Ohio University, chenj@ohio.edu

\section{Funding Sources}

The research reported in this publication was supported by the National Human Genome Research Institute of the National Institutes of Health under Award Number R15HGoog972. The content is solely the responsibility of the authors and does not necessarily represent the official views of the National Institutes of Health.

\section{Notes}

\section{ACKNOWLEDGMENT}

Chen thanks his family, Ohio University, and colleagues for supports during Covid-19 pandemic.

\section{REFERENCES}

(1) Bian, X.; Kim, C.; Karniadakis, G. E. 111 Years of Brownian Motion. Soft Matter 2016, 12, 6331-6346.

(2) Mejlbro, L. The Complete Solution of Fick's Second Law of Diffusion with Time-Dependent Diffusion Coefficient and Surface Concentration. Durab. Concr. Saline Environ. 1996, 25, 127.

(3) Latour, L. L.; Svoboda, K.; Mitra, P. P.; Sotak, C. H. Time-Dependent Diffusion of Water in a Biological Model System. Proc. Natl. Acad. Sci. U.S.A. 1994, 91, 1229-1233.

(4) Weigel, A. V; Simon, B.; Tamkun, M. M.; Krapf, D. Ergodic and Nonergodic Processes Coexist in the Plasma Membrane as Observed by Single-Molecule Tracking. Proc. Natl. Acad. Sci. U.S.A. 2o11, 108, $6438-6443$.

(5) Alhadid, Y.; Chung, S.; Lerner, E.; Taatjes, D. J.; Borukhov, S.; Weiss, S. Studying Transcription Initiation by RNA Polymerase with Diffusion-Based Single-Molecule Fluorescence. Protein Sci. 2017, 
$26,1278-1290$.

(6) Schmidt, T.; Schütz, G. J.; Baumgartner, W.; Gruber, H. J.; Schindler, H. Imaging of Single Molecule Diffusion. Proc. Natl. Acad. Sci. U.S.A. 1996, 93, 2926-2929.

(7) Cooper, J. T.; Harris, J. M. Imaging Fluorescence-Correlation Spectroscopy for Measuring Fast Surface Diffusion at Liquid/Solid Interfaces. Anal. Chem. 2014, 86, 7618-7626.

(8) Kisley, L.; Chen, J.; Mansur, A. P.; Shuang, B.; Kourentzi, K.; Poongavanam, M.-V.; Chen, W.-H.; Dhamane, S.; Willson, R. C.; Landes, C. F. Unified Superresolution Experiments and Stochastic Theory Provide Mechanistic Insight into Protein Ion-Exchange Adsorptive Separations. Proc. Natl. Acad. Sci. U.S.A. 2014, 111, 2075-2080.

(9) Kim, C.; Borodin, O.; Karniadakis, G. E. Quantification of Sampling Uncertainty for Molecular Dynamics Simulation: Time-Dependent Diffusion Coefficient in Simple Fluids. J. Comput. Phys. 2015, 302, 485-508.

(10) Shea, L. D.; Omann, G. M.; Linderman, J. J. Calculation of Diffusion-Limited Kinetics for the Reactions in Collision Coupling and Receptor Cross-Linking. Biophys. J. 1997, 73, 2949-2959.

(11) Zhou, X.; Andoy, N. M.; Liu, G.; Choudhary, E.; Han, K. S.; Shen, H.; Chen, P. Quantitative SuperResolution Imaging Uncovers Reactivity Patterns on Single Nanocatalysts. Nat. Biotechnol. 2o12, 7, 237-241.

(12) Hendriks, F. C.; Meirer, F.; Kubarev, A. V; Ristanović, Z.; Roeffaers, M. B. J.; Vogt, E. T. C.; Bruijnincx, P. C. A.; Weckhuysen, B. M. Single-Molecule Fluorescence Microscopy Reveals Local Diffusion Coefficients in the Pore Network of an Individual Catalyst Particle. J. Am. Chem. Soc. 2017, 139, $13632-13635$

(13) Balke, N.; Jesse, S.; Morozovska, A. N.; Eliseev, E.; Chung, D. W.; Kim, Y.; Adamczyk, L.; Garcia, R. E.; Dudney, N.; Kalinin, S. V. Nanoscale Mapping of Ion Diffusion in a Lithium-Ion Battery Cathode. Nat. Nanotechnol. 2010, 5, 749.

(14) Landes, C. F.; Rambhadran, A.; Taylor, J. N.; Salatan, F.; Jayaraman, V. Structural Landscape of 
Isolated Agonist-Binding Domains from Single AMPA Receptors. Nat. Chem. Biol. 2o11, 7, 168-173.

(15) Giddings, J. C.; Eyring, H. A Molecular Dynamic Theory of Chromatography. J. Phys. Chem. 2oo2, $59,416-421$.

(16) Saltzman, W. M.; Langer, R. Transport Rates of Proteins in Porous Materials with Known Microgeometry. Biophys. J. 1989, 55, 163.

(17) Kärger, J.; Ruthven, D. M. Diffusion in Nanoporous Materials: Fundamental Principles, Insights and Challenges. New J. Chem. 2016, 40, 4027-4048.

(18) Xu, X.-H.; Yeung, E. S. Direct Measurement of Single-Molecule Diffusion and Photodecomposition in Free Solution. Science 1997, 275, 1106 LP - 1109.

(19) Widengren, J.; Rigler, R. Fluorescence Correlation Spectroscopy as a Tool to Investigate Chemical Reactions in Solutions and on Cell Surfaces. Cell. Mol. Biol. (Noisy-le-grand). 1998, 44, 857-879.

(20) Wang, D.; Wu, H.; Schwartz, D. K. Three-Dimensional Tracking of Interfacial Hopping Diffusion. Phys. Rev. Lett. 2017, 119, 268001.

(21) Van Hove, L. Correlations in Space and Time and Born Approximation Scattering in Systems of Interacting Particles. Phys. Rev. 1954, 95, 249.

(22) Wu, H.; Schwartz, D. K. Nanoparticle Tracking to Probe Transport in Porous Media. Acc. Chem. Res. 2020, DOI: 10.1021/acs.accounts.oco0408.

(23) Edward, J. T. Molecular Volumes and the Stokes-Einstein Equation. J. Chem. Educ. 1970, 47, 261.

(24) Mörters, P.; Peres, Y. Brownian Motion; Cambridge University Press, 2010; Vol. 30.

(25) Ward, A. F. H.; Tordai, L. Time-dependence of Boundary Tensions of Solutions I. The Role of Diffusion in Time-effects. J. Chem. Phys. 1946, 14, 453-461.

(26) Langmuir, I.; Schaefer, V. J. The Effect of Dissolved Salts on Insoluble Monolayers. J. Am. Chem. Soc. 1937, 59, 2400-2414.

(27) Li, X.; Shaw, R.; Evans, G. M.; Stevenson, P. A Simple Numerical Solution to the Ward-Tordai Equation for the Adsorption of Non-Ionic Surfactants. Comput. Chem. Eng. 2o10, 34, 146-153. 
(28) Hristov, J. A Unified Nonlinear Fractional Equation of the Diffusion-Controlled Surfactant Adsorption: Reappraisal and New Solution of the Ward-Tordai Problem. J. King Saud Univ. - Sci. 2016, 28, 7-13.

(29) Deshmukh, O. S.; van den Ende, D.; Stuart, M. C.; Mugele, F.; Duits, M. H. G. Hard and Soft Colloids at Fluid Interfaces: Adsorption, Interactions, Assembly \& Rheology. Adv. Colloid Interface Sci. 2015, 222, $215^{-227}$

(30) Baret, J. F. Kinetics of Adsorption from a Solution. Role of the Diffusion and of the AdsorptionDesorption Antagonism. J. Phys. Chem. 1968, 72, 2755-2758.

(31) Liu, J.; Messow, U. Diffusion-Controlled Adsorption Kinetics at the Air/Solution Interface. Colloid Polym. Sci. 2000, 278, 124-129.

(32) Liu, J.; Li, P.; Li, C.; Wang, Y. Diffusion-Controlled Adsorption Kinetics of Aqueous Micellar Solution at Air/Solution Interface. Colloid Polym. Sci. 2009, 287, 1083-1088.

(33) Nguyen, A. V; Phan, C. M.; Evans, G. M. Effect of the Bubble Size on the Dynamic Adsorption of Frothers and Collectors in Flotation. Int. J. Miner. Process. 2006, 79, 18-26.

(34) Pyle, J. R. J. R.; Chen, J. Photobleaching of YOYO-1 in Super-Resolution Single DNA Fluorescence Imaging. Beilstein J. Nanotechnol. 2017, 8, 2296-2306.

（35） Schulten, K.; Kosztin, I. Lectures in Theoretical Biophysics; Citeseer, 200o; Vol. 117.

(36) Shannon, J. D. A Gaussian Moment-Conservation Diffusion Model. J. Appl. Meteorol. 1979, 18, 14061414 .

(37) Klages, R.; Dorfman, J. R. Simple Maps with Fractal Diffusion Coefficients. Phys. Rev. Lett. 1995, 74, 387.

(38) Chen, W.; Sun, H.; Zhang, X.; Korošak, D. Anomalous Diffusion Modeling by Fractal and Fractional Derivatives. Comput. Math. with Appl. 2010, 59, 1754-1758.

(39) Kopelman, R. Fractal Reaction Kinetics. Science 1988, 241, 1620-1626.

(40) Chen, J.; Pyle, J. R.; Sy Piecco, K. W.; Kolomeisky, A. B.; Landes, C. F. A Two-Step Method for 
SmFRET Data Analysis. J. Phys. Chem. B 2016, 120.

(41) Kienle, D. F.; Schwartz, D. K. Complex Salt Dependence of Polymer Diffusion in Polyelectrolyte Multilayers. J. Phys. Chem. Lett. 2019, 10, 987-992.

(42) Laage, D.; Hynes, J. T. A Molecular Jump Mechanism of Water Reorientation. Science 2oo6, 311,832 LP -835 .

(43) Vicente, J. R.; Rafiei Miandashti, A.; Sy Piecco, K. W. E.; Pyle, J. R.; Kordesch, M. E.; Chen, J. SingleParticle Organolead Halide Perovskite Photoluminescence as a Probe for Surface Reaction Kinetics. ACS Appl. Mater. Interfaces 2019, 11, 18034-18043.

(44) Chen, J.; Ruther, R. E.; Tan, Y.; Bishop, L. M.; Hamers, R. J. Molecular Adsorption on ZnO(10-10) Single-Crystal Surfaces: Morphology and Charge Transfer. Langmuir 2012, 28, 10437-10445.

(45) Metropolis, N.; Rosenbluth, A. W.; Rosenbluth, M. N.; Teller, A. H. Equation of State Calculations by Fast Computing Machines. J. Chem. Phys. 1953, 21, 1087-1092.

(46) Camillone, N. Diffusion-Limited Thiol Adsorption on the Gold(111) Surface. Langmuir 20o4, 20, 11991206.

(47) Peterlinz, K. A.; Georgiadis, R. In Situ Kinetics of Self-Assembly by Surface Plasmon Resonance Spectroscopy. Langmuir 1996, 12, 4731-4740. 
TOC:

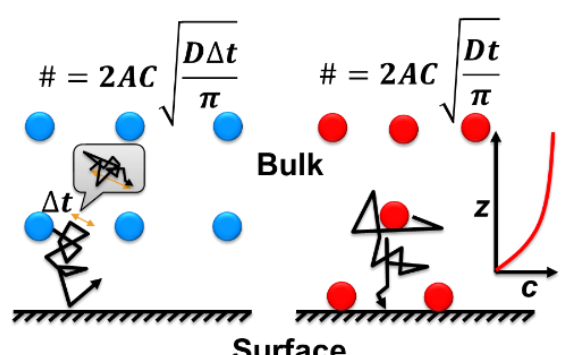

Surface 


\title{
Monte Carlo Simulation of Stochastic Adsorption of Diluted Solute
}

\section{Molecules at an Interface}

\author{
Jixin Chen* \\ Department of Chemistry and Biochemistry, Nanoscale and Quantum Phenomena Institute, Ohio University, Ath- \\ ens, $\mathrm{OH} 45701$.
}

Sample MATLAB Code: 1D control simulation

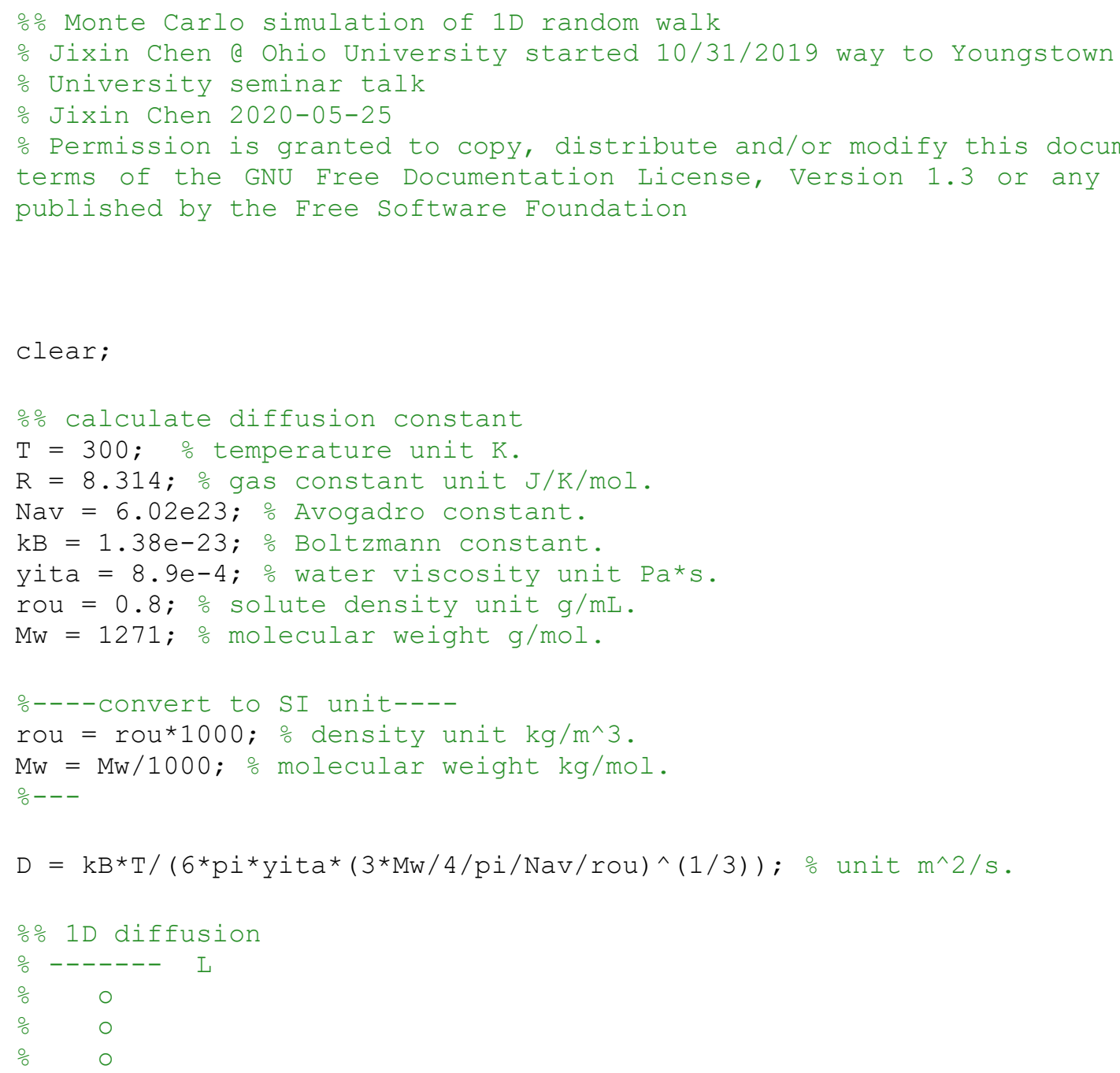


conc $=1 e-6 ; \quad$ concentration unit mole/L.

$\mathrm{L}=100000 ; \%$ space between two surfaces unit nm.

area $=1000 * 2000$; $\frac{\circ}{0}$ surface area of the surface unit $\mathrm{nm}^{\wedge} 2$.

$d t=1 ; \%$ simulated time step unit $\mathrm{ms}$.

lt $=100 ; \%$ total simulated time unit $\mathrm{s}$.

o----convert to SI unit -----

conc $=$ conc*1000*Nav; $\%$ conc unit $m^{\wedge}-3$.

distance $=\operatorname{conc}^{\wedge}(-1 / 3)$; $\frac{\circ}{0}$ unit $m$

distime $=$ distance^2/2/D;

$\mathrm{L}=\mathrm{L} * 1 e-9$; $\frac{\circ}{\circ}$ unit $\mathrm{m}$.

area $=$ area*1e-18; 을 $m^{\wedge} 2$.

$d t=d t * 1 e-3 ;$ ounit $s$.

$\circ----$

\% numm = round (conc*L*area); $\frac{\circ}{\circ}$ number of molecule in the volume.

numm $=10000$;

numt $=$ round $(l t / d t) ; \%$ total time step index.

meandis $=\mathrm{L} /$ numm; $\frac{\circ}{\circ}$ average space between two molecules in $z$.

sigma $=\operatorname{sqrt}\left(2 * D^{*} d t\right) ;$

\% meandisv $=\operatorname{conc}^{\wedge}(-1 / 3)$; $\%$ average space between two molecules in volume.

meantime $=$ meandis^2/2/D; $\frac{\circ}{\circ}$ average time to travel the average space.

\% check meantime and dt. dt should be much smaller ( $>10$ times) than meantime.

display (['meantime/dt $=$ ', num2str(meantime/dt)]);

display (['simu length $=$ ', num2str(lt/dt)]);

display (['num molecules $=$ ', num2str(numm) ]);

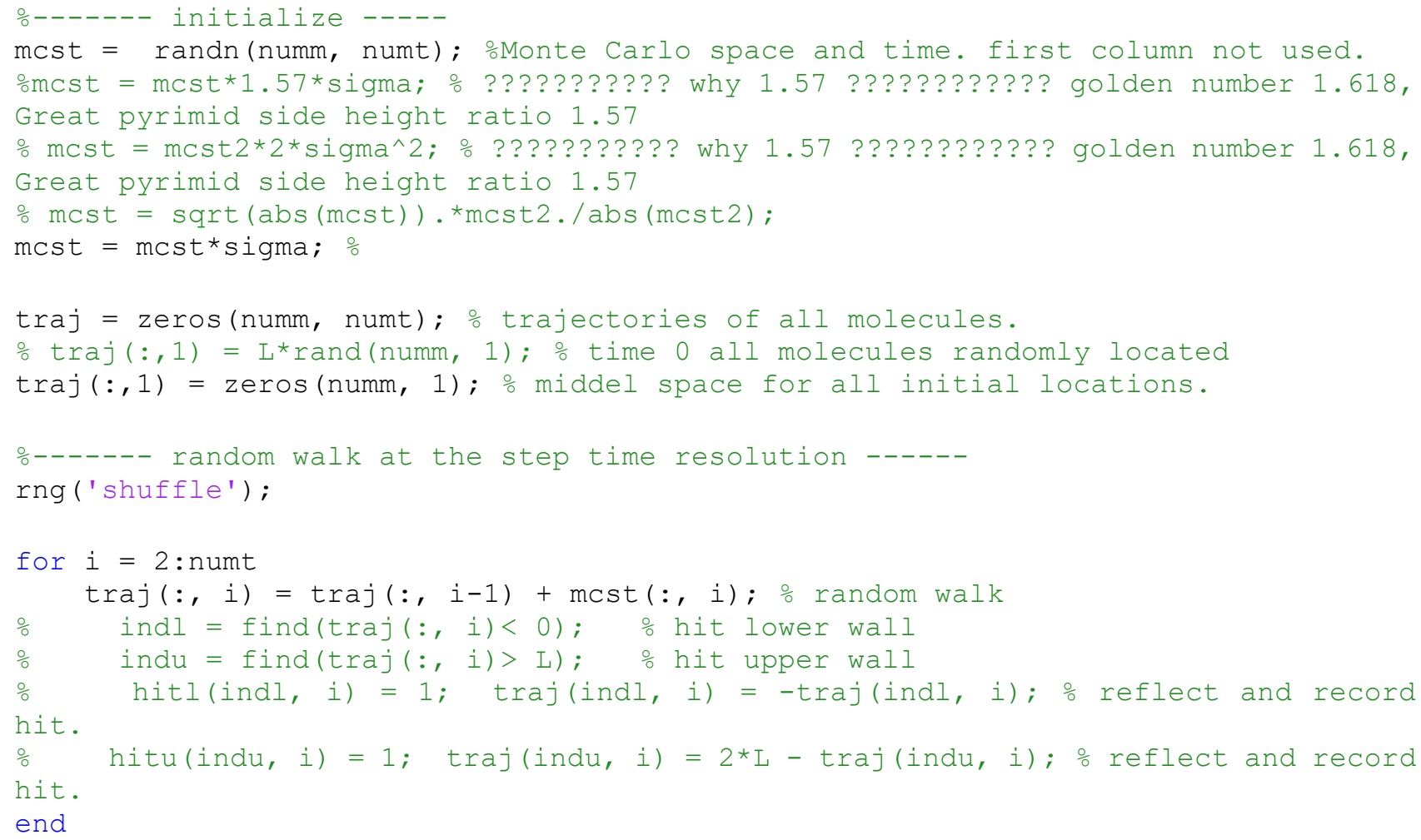




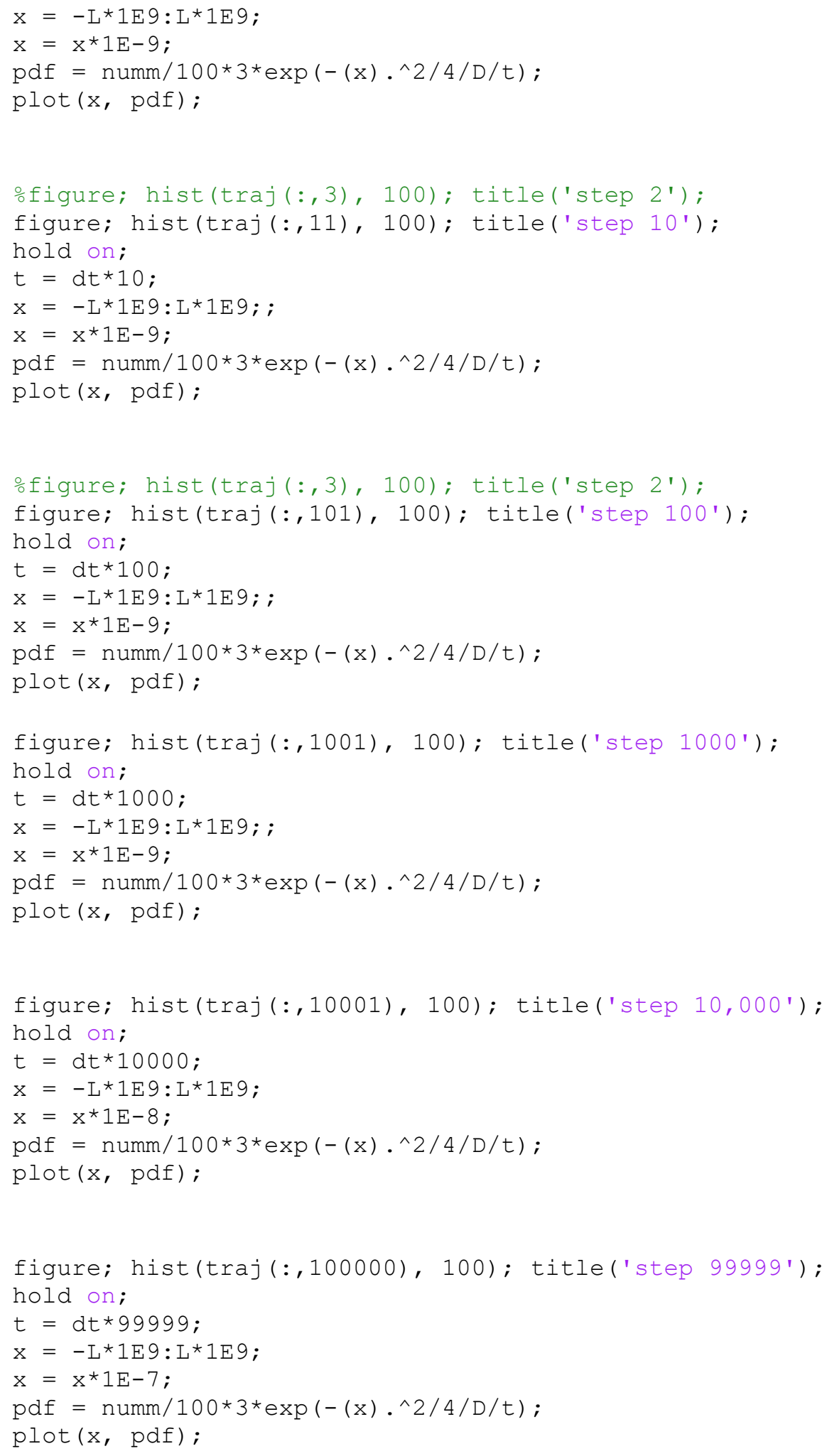

